\title{
A Multi-faceted Educational Approach for Pain Metric Recording Prior to Knee and Hip Arthroplasty: Effects on Documentation by an Acute Pain Service
}

\author{
Alberto Ardon ${ }^{1}$, Matthew Warrick ${ }^{2}$, Tyler Wickas ${ }^{3}$ \\ 1. Anesthesiology, Mayo Clinic, Jacksonville, USA 2. Anesthesiology, University of Florida, Jacksonville, \\ USA 3. Anesthesiology, Florida State University, Tallahassee, USA
}

$\square$ Corresponding author: Alberto Ardon, a.ardon@gmail.com

Disclosures can be found in Additional Information at the end of the article

\section{Abstract}

\section{Background}

Despite the increased use of electronic medical records (EMRs) in past years, the recording of clinically useful baseline pain information may still be lacking. An educational effort targeted at the acute pain service and reinforced by electronic prompting may be an effective way to promote electronic documentation of relevant pain metrics. The objective of this study was to assess whether an educational effort with electronic prompting in the EMR promotes the documentation of baseline pain scores and preoperative opioid use by an acute pain service (APS).

\section{Methods}

A total of 98 patients were included in this study: 49 in the study group and 49 in the control group. The study group consisted of patients who underwent knee and hip arthroplasties after the institution of a multimodal analgesia educational program that also incorporated an electronic prompt to promote behavior change. Primary outcomes were the frequency of documentation of baseline pain scores and preoperative opioid use.

\section{Results}

After the implementation of the education initiative, $67 \%$ of the patients had baseline pain scores recorded in the preoperative APS documentation, compared to $20 \%$ in the control group $(p=0.0001)$. Preoperative opioid use was recorded in $24 \%$ of APS documentation within the control group, but this increased to $73 \%$ after the educational intervention $(p=0.0001)$.

Received 06/14/2019

Review began $06 / 22 / 2019$ Review ended 06/23/2019 Published 06/28/2019

\section{(c) Copyright 2019}

Ardon et al. This is an open access article distributed under the terms of the Creative Commons Attribution License CC-BY 3.0., which permits unrestricted use, distribution, and reproduction in any medium, provided the original author and source are credited.
Documentation of resting pain scores on the day of surgery also increased from $59 \%$ to $87 \%(p=$ 0.0014).

\section{Conclusions}

The introduction of a multi-dimensional educational effort focused on baseline pain metric recording within the context of an analgesic change of practice increased assessment of both baseline pain and preoperative opioid use by APS. These results can be applied to other settings in which a focused change of practice is required and an electronic medical record already utilized.

How to cite this article

Ardon A, Warrick M, Wickas T (June 28, 2019) A Multi-faceted Educational Approach for Pain Metric Recording Prior to Knee and Hip Arthroplasty: Effects on Documentation by an Acute Pain Service. Cureus 11(6): e5030. DOI 10.7759/cureus.5030 
Categories: Anesthesiology

Keywords: education, electronic prompt, analgesic documentation, acute pain service, multimodal analgesia, arthroplasty

\section{Introduction}

The presence of electronic medical records (EMRs) has become almost ubiquitous over the course of the past decade. Specific to perioperative medicine, EMRs have become integral to accurate documentation of patient vital signs and medication administration, as well as capable data repositories and tools to investigate perioperative outcomes. Despite these advantages, an EMR system is only as useful as the data contained therein and the initial input of a patient's relevant health variables is critical. Several recent investigations suggest that prompts in the EMR encouraging healthcare providers to record a particular data point may be influential on behavior and may even have a role in the accuracy and reducing documentation errors [1-4].

Within the realm of perioperative pain management, the accurate recording of analgesic metrics such as pain scores, functional status, and opioid use is critical to providing personalized and effective analgesic interventions. In the context of postoperative analgesia, an important data point that is sometimes overlooked or not recorded in preoperative documentation is a patient's baseline pain score. Some evidence suggests that the higher the patient's preoperative pain level, the more likely the patient will have postoperative pain that will be difficult to control or will develop chronic pain [5-7]. Furthermore, for extremity surgery such as arthroplasty, this baseline pain score should be specific to the operative site to contextualize postoperative pain scores and should include both static and active parameters. Likewise, qualitative and quantitative knowledge of a patient's preoperative opioid analgesic use, if any, may influence the planning and efficacy of postoperative analgesic efforts [8-10]. Furthermore, if this information is recorded prior to surgery instead of after, the risk of recall bias (error caused by the inaccurate recollection of information by study participants) is decreased [11-12]. Recording of this surgery-specific baseline pain data by anesthesia providers in a consistent manner may be challenging. At our institution, approximately less than $25 \%$ of elective arthroplasty patients had this information recorded in the EMR. However, an educational effort targeted at involved providers and reinforced by electronic prompting may be an effective way to promote electronic documentation of these metrics. Such an educational program can be provided as part of the implementation of a multimodal analgesic practice guideline for hip and knee arthroplasties. The primary objective of this study is to assess whether a multi-faceted educational effort promotes the documentation of baseline pain scores and preoperative opioid use by an acute pain service (APS).

\section{Materials And Methods}

The University of Florida - Jacksonville Institutional Review Board approved this study. In this retrospective chart review, the two study groups were:

- Intervention group: consisted of patients undergoing total hip or knee arthroplasties after August 3, 2015, the first surgical date after the educational effort and implementation of the multimodal analgesic guideline.

- Control group: an equivalent cohort matched for age, American Society of Anesthesiologists (ASA) classification, and surgery type who underwent hip or knee arthroplasty prior to August 3, 2015.

To increase the homogeny between the two study groups, exclusion criteria for initial review were age less than 18 years, bilateral surgery, fracture as preoperative surgical diagnosis, placement of antibiotic spacer, and preoperative patient refusal for regional anesthesia. 
A total of 152 patient charts were reviewed for this study. In total, 54 patients were excluded secondary to administration of one or more of the non-opioid analgesics (in the control group), clinical contraindication to one or more of the non-opioid analgesics or the peripheral nerve block. A total of 98 patients were therefore included in this study. A chart review consisted of recording the following information: age, gender, American Society of Anesthesiologists (ASA) score, site of surgery, whether primary or revision surgery, documentation of preoperative baseline pain score and opioid use, regional anesthetic technique employed, administration of acetaminophen, celecoxib, and gabapentin, and postoperative static and active pain scores.

\section{Pre-implementation education}

As a component of the multimodal arthroplasty guideline, all clinical members of the anesthesia APS (eight faculty, one fellow, 12 residents, and one acute pain nurse practitioner) participated in a pre-implementation educational program. With regard to the current study, the purpose of this educational effort was to introduce and reinforce the concept and benefits of multimodal analgesia and more surgery-specific pain data recording. Specific aims with regards to the current study were to 1) promote a more thorough preoperative review of patient pain history, 2) encourage documentation of preoperative baseline pain scores and opioid use, and 3) reinforce postoperative pain score recording. The educational effort consisted of three sessions: one grand rounds lecture, one online module, and one separate review session with residents, who would be the primary individuals evaluating patients and documenting outcome metrics (Figure 1).

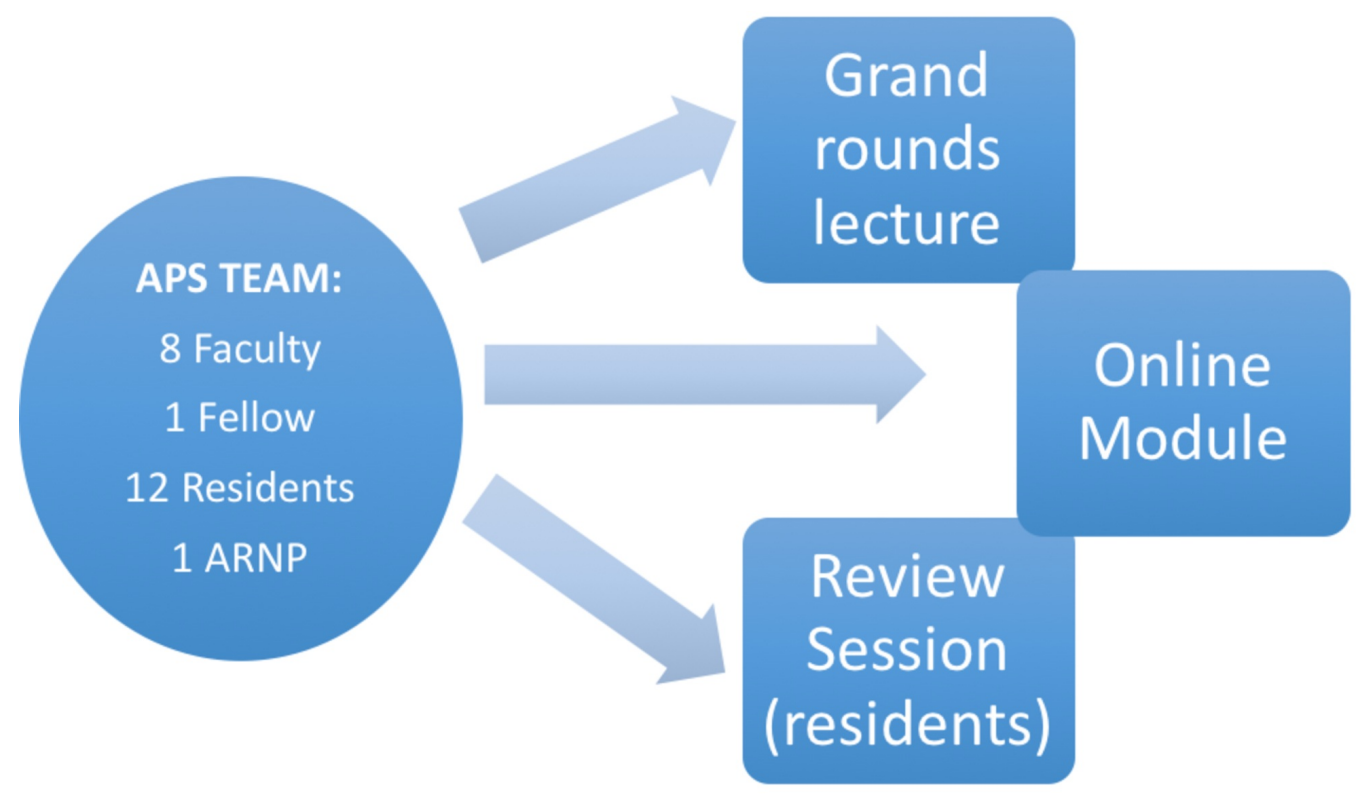

\section{FIGURE 1: Pre-implementation education program components and target audiences}

APS, acute pain service; ARNP, advanced registered nurse practictioner

\section{Multimodal guideline and peri-operative documentation in electronic medical record}

With the introduction of the multimodal analgesic guideline in August 2015 (Table 1), the electronic documentation as filled by the APS was updated to provide electronic prompts for 


\section{Cureus}

baseline pain scores and opioid use (Figure 2).

\begin{tabular}{|c|c|c|c|}
\hline \multirow{6}{*}{$\begin{array}{l}\text { Preoperative } \\
\text { Phase }\end{array}$} & Medications & Dose & Frequency \\
\hline & Acetaminophen & $1000 \mathrm{mg}$ oral & Once \\
\hline & Celecoxib & $400 \mathrm{mg}$ oral & Once \\
\hline & Gabapentin & $600 \mathrm{mg}$ oral & Once \\
\hline & Regional analgesia & & \\
\hline & $\begin{array}{l}\text { Femoral (TKA) or Lumbar Plexus } \\
\text { (THA) nerve block }\end{array}$ & $25-30 \mathrm{ml}$ ropivacaine & \\
\hline \multirow{6}{*}{$\begin{array}{l}\text { Postoperative } \\
\text { Phase }\end{array}$} & Medications & Dose & Frequency \\
\hline & Acetaminophen & $1000 \mathrm{mg}$ oral & Every 6 hours for 48 hours \\
\hline & Celecoxib & $200 \mathrm{mg}$ oral & Every 12 hours for 48 hours \\
\hline & Gabapentin & $600 \mathrm{mg}$ oral & $\begin{array}{l}\text { Every } 12 \text { hours } \times 2 \text { doses, then every } 8 \\
\text { hours for } 24 \text { hours }\end{array}$ \\
\hline & Regional analgesia & & \\
\hline & $\begin{array}{l}\text { Femoral (TKA) or lumbar plexus } \\
\text { (THA) nerve catheter }\end{array}$ & $\begin{array}{l}\text { Bupivacaine } 0.125 \% \text { at } \\
10-12 \mathrm{ml} / \mathrm{hr}\end{array}$ & Continuous until the morning of POD2 \\
\hline TABLE 1: T & KA and THA multimodal & algesic component & \\
\hline \multicolumn{4}{|c|}{ POD, postoperative day; TKA, total knee arthroplasty; THA, total hip arthroplasty } \\
\hline
\end{tabular}




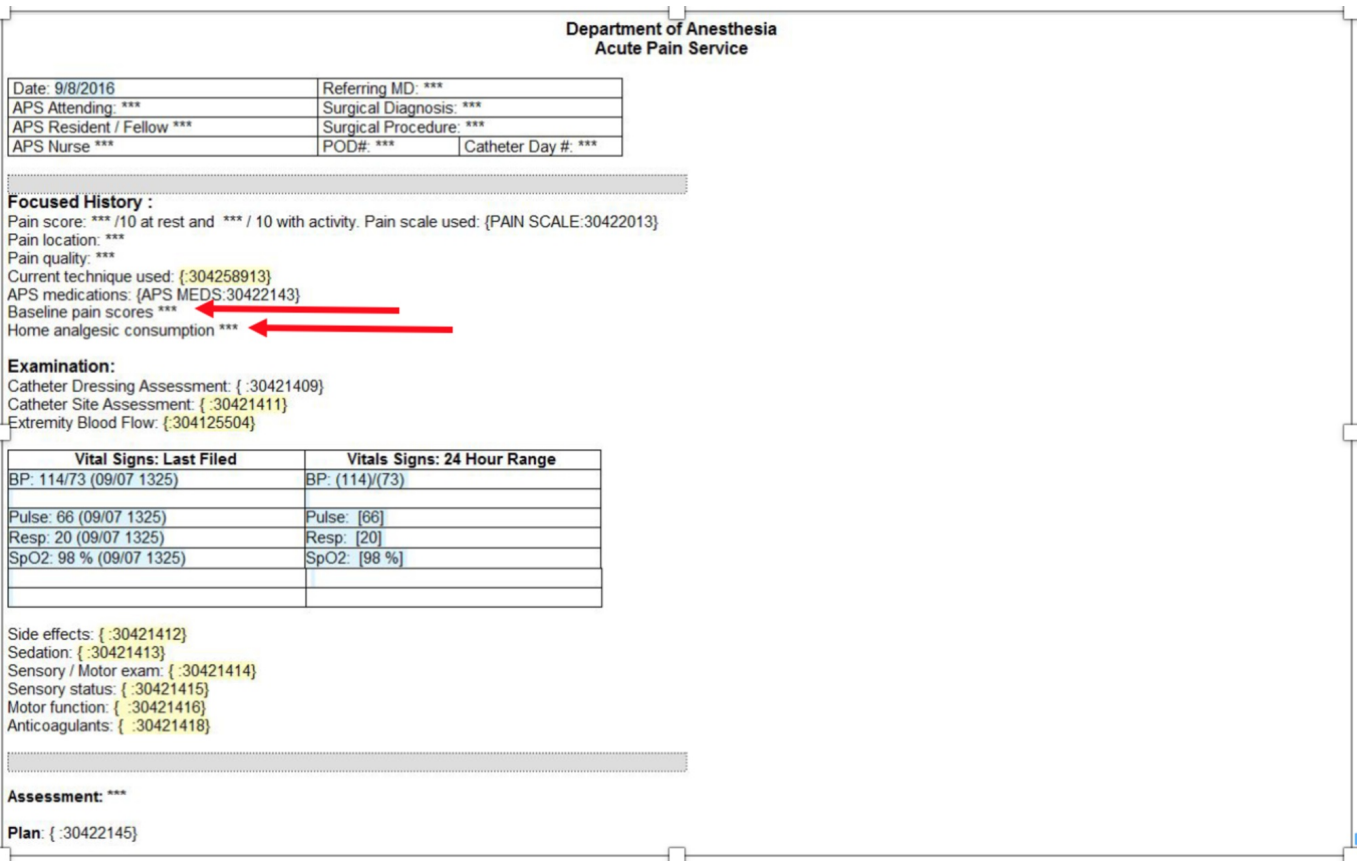

\section{FIGURE 2: Acute pain service consult note}

Arrows point to electronically prompted sections to document baseline pain scores and opioid use.

APS, acute pain service; POD, postoperative day; MD, medical doctor; $\mathrm{BP}$, blood pressure; $\mathrm{SpO}_{2}$, pulse oximetry

During the establishment of this change in the documentation, practitioners were encouraged to record 1) pain scores at the surgical site (at rest and with motion), 2) ambulatory opioid analgesics, if any, and 3) dose and frequency of those analgesics if applicable. These variables were obtained by directly asking patients the following questions:

- On a scale from 0 to 10 ( 0 being no pain and 10 being worst pain imaginable), how much pain do you currently have in your operative knee/hip at rest? With motion?

- During the past week, what opioid medications have you taken for pain on a daily basis?

- What dose of that opioid do you take and how many times a day?

\section{Outcome measures}

Primary outcomes were the frequency of assessment of 1) preoperative pain scores and 2) preoperative ambulatory opioid use as documented in the APS consult note. Preoperative opioid use was defined as a patient taking oral morphine, oxycodone, hydromorphone, methadone, or hydrocodone daily for the week prior to surgery, or a fentanyl transdermal patch for at least one week prior to surgery. Secondary outcomes included frequency of documentation of actual intake of preoperative opioid (usual total dose in a 24-hour period), and frequency of recording of postoperative pain scores (at rest and with motion) by APS on surgical follow-up. Administrations of non-opioid analgesics and peripheral nerve catheter use were also assessed. The use of these multimodal analgesic approaches was considered as consistent with the program guideline if the patient received a peripheral nerve catheter and all three non-opioid analgesics in the preoperative bay and these analgesic methods were 


\section{Cureus}

continued on postoperative day (POD) 1 .

\section{Statistical analysis}

Based on an estimated rate of baseline pain score and preoperative opioid use assessments of approximately $20 \%$ given current clinical practices prior to the establishment of the multimodal approach, we estimated that to detect a 30\% change in incidence (with an alpha of 0.05 and Power of $80 \%$ ), at least 38 patients would be required in each group. Statistical analysis was conducted using AcaStat (AcaStat Software, Poinciana, FL). Two-tailed unpaired $t$-tests were used for quantitative variables; $\chi^{2}$ analysis was utilized for nominal variables.

\section{Results}

Baseline patient characteristics are shown in Table 2. No statistically significant differences existed regarding age, gender, ASA score, or surgery type. Approximately $88 \%$ of patients in the study group underwent a primary arthroplasty, compared to $69 \%$ in the control group $(p=$ 0.027).

\begin{tabular}{|c|c|c|c|c|}
\hline & & Control $(n=49)$ & Study (n = 49) & $P$ value \\
\hline Age & & $57.4(10.1)$ & $61(9.5)$ & 0.07 \\
\hline \multirow[t]{2}{*}{ Gender } & Male & $47 \%$ & $35 \%$ & 0.22 \\
\hline & Female & $53 \%$ & $65 \%$ & \\
\hline ASA (median) & & 3 & 3 & 0.45 \\
\hline \multirow[t]{2}{*}{ Surgery } & TKA & $67 \%$ & $61 \%$ & 0.53 \\
\hline & IHA & $33 \%$ & $39 \%$ & \\
\hline \multirow[t]{2}{*}{ Primary or revision* } & Primary & $69 \%$ & $88 \%$ & 0.027 \\
\hline & Revision & $31 \%$ & $12 \%$ & \\
\hline
\end{tabular}

\section{TABLE 2: Baseline characteristics}

$P$ value $<0.05$ denoted by asterisk $\left({ }^{*}\right)$.

ASA, American Society of Anesthesiologists; TKA, total knee arthroplasty; THA, total hip arthroplasty

\section{Primary outcomes}

Primary outcomes are shown in Table 3. Approximately 20\% of patients in the control group had baseline pain scores recorded in the preoperative APS documentation, compared to $67 \%$ in the study group $(p=0.0001)$. Of those patients whose baseline pain score was assessed, there was no significant difference in the baseline pain score before $(7.70 \pm 1.89)$ and after $(7.52 \pm$ $1.54)$ multimodal guideline implementation $(p=0.75)$. Preoperative opioid use was recorded in $24 \%$ of APS documentation within the control group, but this percentage increased to $73 \%$ after the educational intervention $(p=0.0001)$. 


\section{Cureus}

\begin{tabular}{|c|c|c|c|c|}
\hline & & $\begin{array}{l}\text { Control } \\
(n=49)\end{array}$ & $\begin{array}{l}\text { Study(n } \\
=49)\end{array}$ & $\begin{array}{l}P \\
\text { value }\end{array}$ \\
\hline Baseline pain score assessed?* & Yes & $20 \%$ & $67 \%$ & 0.0001 \\
\hline Baseline pain score (if available) & & $\begin{array}{l}7.70 \\
(1.89)\end{array}$ & $\begin{array}{l}7.52 \\
(1.54)\end{array}$ & 0.75 \\
\hline Preoperative opioid use assessed?* & Yes & $24 \%$ & $73 \%$ & 0.0001 \\
\hline Preoperative opioid use (if available) & Yes & $83 \%$ & $69 \%$ & 0.35 \\
\hline $\begin{array}{l}\text { Among those whose pre-op opioid use was assessed as "yes", what } \\
\text { percentage had actual opioid intake documented?* }\end{array}$ & & $68 \%$ & $20 \%$ & 0.0001 \\
\hline \multirow[t]{2}{*}{ Static pain score recorded by APS* } & POD 0 & $59.2 \%$ & $87 \%$ & 0.0014 \\
\hline & POD 1 & $79.6 \%$ & $91.8 \%$ & 0.08 \\
\hline \multirow[t]{2}{*}{ Active pain score recorded by APS } & POD 0 & $28.6 \%$ & $18.4 \%$ & 0.23 \\
\hline & POD 1 & $59.2 \%$ & $40.8 \%$ & 0.225 \\
\hline \multirow[t]{3}{*}{ Use of non-opioid analgesics } & Acetaminophen & & $100 \%$ & \\
\hline & Celecoxib & & $88 \%$ & \\
\hline & Gabapentin & & $98 \%$ & \\
\hline \multirow[t]{4}{*}{ Regional analgesic technique } & Catheter & $82 \%$ & $92 \%$ & 0.09 \\
\hline & Single shot & $8 \%$ & $6 \%$ & \\
\hline & $\begin{array}{l}\text { Intrathecal } \\
\text { opioid }\end{array}$ & 0 & $2 \%$ & \\
\hline & Failed block & $10 \%$ & 0 & \\
\hline
\end{tabular}

\section{TABLE 3: Primary and secondary outcomes}

$P$ value $<0.05$ denoted by asterisk $\left({ }^{*}\right) ;$ APS, acute pain service; POD, postoperative day

\section{Secondary outcomes}

Secondary outcomes are also shown in Table 3. Of the patients who did have preoperative opioid use assessed, $83 \%$ of control group patients used preoperative opioids versus $69 \%$ of study patients $(p=0.35)$. Among these patients, $68 \%$ of control patients had 24 -hour preoperative opioid intake documented, compared to only $20 \%$ of study patients $(p=0.0001)$. Regarding documentation of POD 0 and POD 1 resting and active pain scores by APS, only documentation of resting pain scores on POD 0 resulted in a statistically significant difference between the two groups ( $59 \%$ vs $87 \%, p=0.0014$ ). Use of multimodal analgesics was $100 \%$ for acetaminophen, $88 \%$ for celecoxib, and $98 \%$ for gabapentin after the introduction of the analgesic guidelines. Peripheral nerve catheter use (rather than single shot nerve blockade) did not increase significantly ( $82 \%$ vs $92 \%, p=0.09$ ). Approximately $10 \%$ of patients in the control 


\section{Cureus}

group were found to have no sensory deficit in the recovery area and thus were classified as "failed block".

As more patients in the protocol group underwent primary arthroplasty, outcomes were reexamined within this sub-population (Table 4). Rates of baseline pain score and opioid use assessment were higher for study patients (24\% vs $67 \%, p=0.0001 ; 30 \%$ vs $74 \%, p=0.0004$, respectively), consistent with general results.

\begin{tabular}{|c|c|c|c|c|}
\hline & & Control (n = 34) & Study $(n=43)$ & $P$ value \\
\hline Baseline Pain Score Assessed?* & Yes & $24 \%$ & $67 \%$ & 0.0001 \\
\hline Baseline pain score (if available) & & 7.25 (1.83) & 7.52 (1.55) & 0.68 \\
\hline Preoperative opioid use assessed?* & Yes & $30 \%$ & $74 \%$ & 0.0004 \\
\hline
\end{tabular}

\section{TABLE 4: Primary outcome measures controlling for primary arthroplasty patients}

$P$ value $<0.05$ denoted by asterisk $\left(^{*}\right)$

\section{Discussion}

In our study, the use of a multifaceted educational program that included didactic instruction and electronic prompting emphasizing documentation of baseline pain characteristics in the EMR was associated with a significant increase in recording of both baseline pain scores and opioid use. This finding is in agreement with previous research which suggests that both educational efforts and electronic prompting are effective in causing behavior change [1-4,13]. It is also in agreement with studies that suggest that behavior change is more likely when a practical application is included within the educational framework [14-16]. To our knowledge, however, this is the first study to demonstrate these effects within the context of an APS. Clinical educational efforts may have an ultimate higher rate of success when adapted to the local clinical framework and accompanied by active reinforcement such as simulation or teaching [17-19]. Clinical guidelines, which encourage target activities and promote practices learned in above educational efforts, may be advantageous to translate knowledge gain into internalized behavior change. While documentation of baseline pain or opioid use was encouraged in this project via education and facilitated electronically, it was not made compulsory so as to not introduce bias. In other words, the APS consult note could be completed without this baseline pain information. Thus, clinicians who, after the introduction of this change in the documentation, recorded the information did so after a visual electronic prompt which could have been bypassed if wanted. Thus, we believe that the observed increase in frequency in the documentation of baseline pain scores and preoperative opioid use was indeed secondary to the intervention. The implication of this work is that a simple educational intervention, coupled with a behavior change prompt, can alter clinician behavior and promote better documentation. Although our study examined this behavior change purely within the framework of an APS, the concept could be applied across different clinical contexts and learner populations.

With regards to our patient population, the establishment of this multimodal analgesic guideline: 1) revealed that patients undergoing arthroplasties at our institution had a fairly high baseline pain score (>7/10), and 2) suggest that almost three-quarters of our hip and knee arthroplasty patient population may use opioid medication on a regular basis prior to surgery. 
Knowledge of these high preoperative pain scores and rates of use of opioid analgesics is very useful for the analgesic management of patients after major surgery such as arthroplasty for two reasons. First, the accurate assessment of preoperative pain scores provides a relative framework within which to build both clinician and patient expectations, which can affect postsurgical outcome [12]. A patient who, for example, has a numeric baseline knee pain score of 8 out of 10 should be counseled that they may be at increased risk of postoperative pain that may be more difficult to control. Second, identification of patients who have significant recent or current exposure to opioids prior to surgery allows the clinician to assess the risk of opioid tolerance and adjust the anesthetic and analgesic plan accordingly, given recent evidence that exposure to opioids may indeed alter anesthetic/analgesic requirements [20].

Despite the improvement in recording the baseline preoperative opioid use, the recording of the actual amount of preoperative opioid analgesic consumed by patients in a 24-hour period did not improve and in fact worsened after the educational intervention ( $68 \%$ vs $20 \%, p<0.001$ ). The reasons for this decrease in recording of this information are unclear but may be secondary to 1$)$ a significantly smaller number of patients $(n=10)$ that were confirmed to be using opioids preoperatively in the control group compared to those in the intervention group $(n=25) ; 2)$ a need for further, more targeted educational reinforcement for obtaining this particular parameter or 3) an overall need for deeper cultural change with respect to the significance of this data. We believe that obtaining a relative idea of the amount of opioid, if any, that a patient is consuming prior to undergoing a major operation is important as this variable can influence the patient's postoperative risk profile as well as directly affect intraoperative and postoperative analgesia.

Although the recording of static pain scores by APS on POD 0 increased by $28 \%$, the recording of active pain scores did not change significantly on either POD 0 or POD 1. Differentiating between static and active pain may be critical in establishing appropriate analgesia to aid in physical therapy. Thus, it is concerning that this documentation did not increase. However, in retrospect, the recording of these specific parameters could have been addressed more thoroughly in the educational curriculum. More education may be required, as clinician biases may influence behavior and decrease the impact of clinical documentation changes [21]. Consequently, educational materials at our institution now include an emphasis on differentiating and recording both static and active pain, which may also be applicable to other institutions as the use of active/functional pain scores increases in clinical practice overall.

\section{Limitations}

Some limitations exist in our study. First, despite an increase in the documentation of whether or not a patient was taking preoperative analgesics, documentation of the actual daily dosage prior to surgery was poor after implementation of the protocol. In fact, the rate of recording of this variable decreased by $48 \%$. We suspect the poor recording of this variable suggests a need for either an increased educational emphasis on this variable or an electronic prompt that first asks if the patient has preoperative opioid use and, if the response is yes, thereafter asks the clinician document the 24-hour intake. Without knowledge of the extent of preoperative opioid exposure, assessing relative postoperative opioid requirements may be very difficult. Likewise, as tolerance is defined as the use of $60 \mathrm{mg}$ of oral morphine equivalents per day for at least seven days whether the patient is actually opioid tolerant or the degree to which the patient is at risk for opioid-related side effects immediately postoperatively can be unknown or at the very least subject to recall bias if this information is not obtained prior to surgery [22]. Indeed, some research suggests that opioid-tolerant patients are in fact at increased risk of respiratory depression compared to an opioid-naïve population [23].

Second, an issue that deserves consideration is whether the observed changes in behavior were secondary to factors outside the boundaries of the study. Specifically, 1) had clinicians been 
exposed to any other educational interventions on the same topic previously? and 2) did social pressure rather than the intervention cause a behavior change? For the first point, no other concurrent educational intervention or hospital policy was implemented or ongoing six months prior to or during the implementation of our educational/clinical protocol. While it may be possible that some clinicians had previously had some education on this topic, the use of multimodal analgesia and a comprehensive peri-operative pain documentation approach was not commonplace in our practice, and thus any previous exposure is unlikely to have influenced the observed results. Regarding social pressure, while this is certainly a possibility, we believe that such social pressure would arise as a consequence of the study intervention, and thus still be attributed to the educational/electronic prompting system. Therefore, we believe that overall the change in behavior as indicated by an increase in the documentation was, in fact, a result of the efforts described in this study.

Lastly, the reason for a 10\% incidence of "failed block" in the control group (compared to 0\% in the study group) is unclear. We speculate that the decrease in block failure after the study intervention was related to an educational component that encouraged assessing sensory deficit soon after peripheral nerve blockade, but this explanation assumes that no other change in clinical practice occurred.

\section{Conclusions}

In conclusion, the introduction of a multi-dimensional educational effort for the implementation of a multimodal analgesic guideline increased assessment of both baseline pain and preoperative opioid use by the acute pain service. These results can be applied to other settings in which a focused change of practice is required and an electronic medical record already utilized. Future studies should be done to further examine and prospectively verify and supplement these results.

\section{Additional Information \\ Disclosures}

Human subjects: Consent was obtained by all participants in this study. University of Florida College of Medicine Jacksonville Institutional Review Board issued approval 201600034. This report describes human research. IRB contact information: University of Florida College of Medicine - Jacksonville Institutional Review Board. 9th Floor, Tower II, 580 West 8th Street, Jacksonville, FL 32209 Study ID IRB 201600034 Phone: 904-244-9478 This study was conducted with written informed consent from the study subjects. . Animal subjects: All authors have confirmed that this study did not involve animal subjects or tissue. Conflicts of interest: In compliance with the ICMJE uniform disclosure form, all authors declare the following:

Payment/services info: All authors have declared that no financial support was received from any organization for the submitted work. Financial relationships: All authors have declared that they have no financial relationships at present or within the previous three years with any organizations that might have an interest in the submitted work. Other relationships: All authors have declared that there are no other relationships or activities that could appear to have influenced the submitted work.

\section{References}

1. Haberman S, Feldman J, Merhi ZO, Markenson G, Cohen W, Minkoff H: Effect of clinicaldecision support on documentation compliance in an electronic medical record. Obstetrics \& Gynecology. 2009, 114:311-17. 10.1097/AOG.0b013e3181af2cb0

2. Patel MS, Volpp KG, Small DS, et al.: Using active choice within the electronic health record to increase physician ordering and patient completion of high-value cancer screening tests. Healthcare. 2016, 4:340-45. 10.1016/j.hjdsi.2016.04.005 
3. Ruffin MT, Plegue MA, Rockwell PG, Young AP, Patel DA, Yeazel MW: Impact of an electronic health record reminder on human papillomavirus vaccine initiation and timely completion. JABFM. 2015, 28:324-333. 10.3122/jabfm.2015.03.140082

4. Sandberg WS, Sandberg EH, Seim AR, Anupama S, Ehrenfeld JM, Spring SF, Walsh JL: Realtime checking of electronic anesthesia records for documentation errors and automatically text messaging clinicians improves quality of documentation. Anesth Analg. 2008, 106:192201. 10.1213/01.ane.0000289640.38523.bc

5. Lutz C, Baverel L, Colombet P, et al.: Pain after outpatient vs inpatient ACL reconstruction: French prospective study of 1076 patients. Orthop Traumatol Surg Res. 2016, 102:265-270. 10.1016/j.otsr.2016.08.009

6. Kampe S, Geismann B, Weinreich G, Stamatis G, Ebmeyer U, Gerbershagen HJ: The influence of type of anesthesia, perioperative pain, and preoperative health status on chronic pain six months after thoracotomy - a prospective cohort study. Pain Med. 2016; pii:pnw230. [epub ahead of print, 10.1093/pm/pnw230

7. Brummett CM, Janda AM, Schueller CM, Tsodikov A, Morris M, Williams DA, Clauw DJ: Survey criteria for fibromyalgia independently predict increased postoperative opioid consumption after lower-extremity joint arthroplasty: a prospective, observational cohort study. Anesthesiology. 2013, 119:1434-43. 10.1097/ALN.0b013e3182a8eb1f

8. Sanansilp V, Dejarkom S, Deetayart S: Postoperative pain management and the risk factors in major operation: a baseline study of acute pain service, Siriraj hospital. J Med Assoc Thai. 2016, 99:549-56.

9. Hah JM, Sharifzadeh Y, Wang BM, Gillespie MJ, Goodman SB, Mackey SC, Carroll IR: Factors associated with opioid use in a cohort of patients presenting for surgery. Pain Res Treat. 2015, 2015:829696. 10.1155/2015/829696

10. Morris BJ, Sciascia AD, Jacobs CA, Edwards TB: Preoperative opioid use associated with worse outcomes after anatomic shoulder arthroplasty. J Shoulder Elbow Surg. 2016, 25:619-23. 10.1016/j.jse.2015.09.017

11. Lowe JT, Li X, Fasulo SM, Testa EJ, Jawa A: Patients recall worse preoperative pain after shoulder arthroplasty than originally reported: a study of recall accuracy using the American Shoulder and Elbow Surgeons score. J Shoulder Elbow Surg. 2016, 16:30405-0. 10.1016/j.jse.2016.09.004

12. Gandhi R, Davey JR, Mahomed N: Patient expectations predict greater pain relief with joint arthroplasty. J Arthroplasty. 2009, 24:716-21. 10.1016/j.arth.2008.05.016

13. Saddawi D, Schumacher DJ, Baker KH, Charnin JE, Gollwitzer PM: Changing physician behavior with implementation intentions: closing the gap between intentions and actions. Academic Medicine. 2016, 91:1211-16. 10.1097/ACM.0000000000001172

14. Hariharan S: Ethical issues in anesthesia: the need for a more practical and contextual approach in teaching. J Anesth. 2009, 23:409-12. 10.1007/s00540-009-0776-x

15. Lorello GR, Cook DA, Johnson RL, Brydges R: Simulation-based training in anaesthesiology: a systematic review and meta-analysis. Br J Anaesth. 2014, 112:231-45. 10.1093/bja/aet414

16. Weiss V, Needlman R: To teach is to learn twice. Arch Pediatr Adolesc Med. 1998, 152:190-92. 10.1001/archpedi.152.2.190

17. Dubowitz G, Evans FM: Developing a curriculum for anaesthesia training in low- and middleincome countries. Best Pract Res Clin Anaesthesiol. 2012, 26:17-21. 10.1016/j.bpa.2012.02.004

18. Murray DJ: Current trends in simulation training in anesthesia: a review . Minerva Anestesiol. 2011, 77:528-33.

19. Prober CG, Heath C: Lecture halls without lectures - a proposal for medical education . New Engl J Med. 2012, 366:1657-9. 10.1056/NEJMp1202451

20. Liu QL, Gold MS: Opioid-induced loss of local anesthetic potency in the rat sciatic nerve . Anesthesiology. 2016, 125:755-64. 10.1097/ALN.0000000000001239

21. Zadove P, Plegue MA, Uhlmann WR, Ruffin MT: Prompting primary care providers about increased patient risk as a result of family history: does it work?. J Am Board Fam Med. 2015, 28:334-342. 10.3122/jabfm.2015.03.140149

22. Wenzel JT, Schwenk ES, Baratta JL, Viscusi ER: Managing opioid-tolerant patients in the perioperative surgical home. Anesthesiology Clin. 2016, 34:287-301.

10.1016/j.anclin.2016.01.005

23. Hayhurst CJ, Durieux ME: Differential opioid tolerance and opioid-induced hyperalgesia: a clinical reality. Anesthesiology. 2016, 124:483-488. 10.1097/ALN.0000000000000963 